\title{
Positive Time: Balanced Time Perspective and Positive Orientation
}

\author{
Małgorzata Sobol-Kwapinska ${ }^{1}$ • \\ Tomasz Jankowski ${ }^{1}$
}

Published online: 5 July 2015

(C) The Author(s) 2015. This article is published with open access at Springerlink.com

\begin{abstract}
The aim of the research presented in this article is to investigate the relationship between positive orientation and time perspective as outlined by Zimbardo and Boyd ( $\mathrm{J}$ Pers Soc Psychol 77:1271-1288, 1999) and extended by an additional form of concentration on the present. The Polish version of the Zimbardo Time Perspective Inventory was used. A third type of present time perspective, namely active concentration on the present, was measured by the Carpe Diem Scale. One hundred and eighty five Polish people (non-students) participated in a survey to which a canonical-correlation analysis and a cluster analysis were applied. Positive orientation was correlated with a balanced temporal profile comprising a strong positive past orientation, moderate concentration on the future, poor fatalistic time perspective, poor concentration on the negative past and moderate active concentration on the present. Whereas a positive orientation is based on a general positive attitude towards life and self, a balanced time perspective reflects a general positive attitude towards time, in which an active concentration on the present plays an important role.
\end{abstract}

Keywords Time perspective Positive orientation - Balanced time perspective . Carpe Diem

\section{Introduction}

The question of optimal attitude towards time preoccupies both researchers and also ordinary people trying to pursue happiness in everyday life. Some people believe that happiness means positive memories, while others assume that happiness comes down to achieving goals and planning for the future. There are also people who live according to the principle that only the 'here and now' gives pleasure. One might say that people seek

Małgorzata Sobol-Kwapinska

sobol@kul.pl

1 Department of Personality Psychology, Catholic University of Lublin, al. Raclawickie 14, 20-950 Lublin, Poland 
happiness in different time perspectives (TP). Is it right, however, to attach significance to only one of the time dimensions (the past, the present or the future) and belittle the importance of the other dimensions? In other words, is it sufficient to study the relationships among the various dimensions of TP and a sense of happiness separately from other temporal dimensions? It seems that it is more reliable to analyse a given time dimension in the context of other attitudes towards time. For example, when a deep positive concentration on the past is combined with a deep concentration on the future, its relationship with well-being will be different than if it were combined with a poor concentration on the future. One might assume that an analysis of a person's temporal profile, that is, an analysis of intensity of particular elements of the TP structure, is an important source of knowledge about that person.

\subsection{Time Perspective}

The concept of TP as presented by Zimbardo (Boniwell and Zimbardo 2004; Zimbardo and Boyd 1999, 2008) constitutes the theoretical basis for this study. According to this concept, cognitive processes divide human experience into past, present and future temporal frames (D'Alessio et al. 2003; Nuttin and Lens 1985; Zimbardo and Boyd 1999). When one of these frames affects human behaviour, we refer to TP or temporal orientation (TO) (Ryack 2012; Zimbardo and Boyd 1999). TP is defined as the subjective, often unconscious, manner in which a person refers to time, the process by which the continual flow of personal and social experience is assigned to each temporal category (Boyd and Zimbardo 2005). Zimbardo and Boyd (1999) distinguished five types of TP: concentration on negative past; concentration on positive past; hedonistic concentration on present; fatalistic concentration on present, and concentration on future. People who concentrate on a negatively perceived past often think back to sad moments from the past. They remember mostly unpleasant events, traumas and disappointments from the past. Concentration on positively evaluated events from the past links with thinking with pleasure about the past, paying attention primarily to the good things that have happened and collecting memorabilia from the past. Hedonistic concentration on the pleasure of the 'here and now' is associated with disregard for experiences from the past. Persons with this hedonistic TP often focus on pleasure, regardless of the consequences of their own actions. For them, pleasure is the most important thing in life, even given the risks involved. A fatalistic TP is a passive persistence in the present stemming from a conviction that people are unable to influence the future. People with a fatalistic concentration on the present believe that everything in life depends on destiny, so the only thing left to do is to passively stand in the present. People who are concentrated on future are interested in what will happen and attach significance primarily to planning, formulating and achieving goals. They often think with pleasure about their own future, make plans for it, dream about it and formulate goals for this future (Zimbardo and Boyd 1999, 2008). The fatalistic and hedonistic temporal attitudes do not cover all possible types within the present TP. In our studies we also dealt with a third kind of present TP, the active concentration on the present (Carpe Diem; Sobol-Kwapinska 2009, 2013).

\subsection{Active Concentration on the Present}

This type of TP is defined as full concentration of attention on the 'here and now', combined with perceiving the value of each moment, a unique, one-of-a-kind moment. 
There are two aspects of active concentration on the present: focusing attention on what is happening 'here and now' and the awareness of the value of each moment of life. Such an attitude does not imply negligence of the past and disregard for consequences of the present situation, which is typical of hedonistic orientation. The point is to free oneself from an incriminatory past or from absorbing thoughts about what is to happen in a moment. Focusing attention on the moment allows full presence in the now, which is combined with the feeling of freshness and spontaneity in the perception of reality. Recognising the value and uniqueness of each moment of life often comes from reflection on the transience of life. People with active concentration on the present are able to fully concentrate on the 'here and now'. They like concentrating on the present, and they recognise the value of such attitude towards time (Sobol-Kwapinska 2009, 2013).

\subsection{The Relationship Between the Time Perspective Dimensions and the Quality of Functioning}

The literature abounds with many results that describe the relationship between a specific attitude towards a given dimension of time and the quality of psychological functioning. Most studies focus on the relationship between concentration on the future and various measures of well-being. Wills et al. (2001), using measures relating to a theory of coping with stress, discovered a positive relationship between concentration on the future and a sense of control and positive affect. Similarly, Zaleski et al. (2001) found that having longterm goals correlated positively with various measures of well-being, especially with a sense of meaning in life, a sense of self-efficacy in social relations, and with realism. Concentration on the future correlated with health-oriented behaviours, such as having preventive screenings (Boyd and Zimbardo 2005), optimism (Zimbardo and Boyd 1999) and academic achievements (Barber et al. 2009; Boyd and Zimbardo 2005; Nosal and Bajcar 2004). At the same time, a very deep concentration on the future may entail reduced spontaneity and a relative inability to enjoy the 'here and now' (Boniwell and Zimbardo 2004; Sobol-Kwapinska 2009, 2013; Zimbardo and Boyd 2008).

Recent studies show that the positive past correlates positively with high self-esteem, a sense of security and happiness, having social support, agreeableness and energy (Bryant et al. 2005; Lyubomirsky and Nolen-Hoeksema 1995; Zimbardo and Boyd 1999). Negative past is correlated with depression, anxiety and low self-esteem, in addition to problems with establishing social relations, and various addictions (Klingemann 2001; Lyubomirsky and Nolen-Hoeksema 1995; Nolen-Hoeksema and Morrow 1993; Zimbardo and Boyd 1999).

Concentration on the present, both fatalistic and hedonistic, correlates positively with risky behaviours, such as excessively fast driving (Zimbardo et al. 1997), alcohol consumption and drug abuse (Daugherty and Brase 2010; Keough et al. 1999; Rothspan and Read 1996; Strathman et al. 1994). The results of studies carried out by Sobol-Kwapinska $(2009,2013)$ revealed a significantly positive relationship between active concentration on the present and having a sense of satisfaction with life, a sense of meaning in life, experiencing positive emotions, and perceiving time as creative and friendly.

Summing up this brief review, it seems that a concentration on the positive past and the future as well as an active concentration on the present are dimensions that correlate with the positive aspects of people's functioning, whereas a fatalistic TP and a concentration on a negatively perceived past have particularly negative consequences. 


\subsection{Balanced Time Perspective}

Recent studies on the relationship between TP and well-being focus on issues related to the so-called balanced time perspective (BTP). The importance of temporal balance has been brought up before; for example, Shostrom (1974) used the term 'time competence' as an essential element of a self-updating personality. According to Shostrom, a self-updating person is primarily characterised by time competence and therefore is able to fully live 'here and now'. Such an individual is able to link the past with the future in the present and seems to be less constrained by a sense of guilt, grief and anger from the past, while aspirations are linked with the goals being worked on. Shostrom (1974) emphasised the inadequacy of concentrating on only one of the three dimensions of time. Lennings et al. (1998), applying a canonical analysis, obtained two canonical functions showing independent sets of relationships between the different characteristics of temporal perspective and the selected personality variables. One of them contains an update temporal profile (characterised by a broad future-oriented perspective, positive attitude towards time and a strong sense of the passage of time), while the other contains a set of variables that might be interpreted as an atomistic temporal profile (concentration on the present and the nearest future without integration of the past into the future). People characterised by individual profiles differ from one another, for example in terms of the ability to postpone gratification and self-control (which is higher in the updating profile).

According to Zimbardo and Boyd $(1999,2008)$, BTP is a combination of deep concentration on a positively perceived past, moderate concentration on the future, moderate hedonistic concentration on the present, poor fatalistic concentration on the present, and poor concentration on a negatively perceived past. Drake et al. (2008) studied the connection between BTP and a subjective sense of happiness and mindfulness. They applied quartile deviations in order to distinguish a group of individuals (from all respondents) whose intensity of various temporal scales was consistent with the exemplary profile proposed by Zimbardo. As expected, the respondents characterised by a BTP were happier and demonstrated higher mindfulness compared to those with other profiles. Boniwell et al. (2010) adopted a different approach while investigating relationships between BTP and well-being; they applied a hierarchical cluster analysis to the five scales of the Zimbardo Time Perspective Inventory (ZTPI). Four clusters were selected, of which one best reflected the profile typical for people with a well-balanced TP (moderate concentration on a positive past and future, slightly lower hedonistic concentration, and poor fatalistic concentration, in addition to poor concentration on negative past). The respondents from the BTP group were characterised by greater life satisfaction, less negative affection, frequent positive affection and stronger self-update. In studies conducted with the use of the Russian version of the ZTPI, the group of respondents with a profile similar to BTP, when compared to people from groups of other temporal profiles, was characterised by a stronger sense of meaning in life, greater life satisfaction, stronger subjectively perceived well-being, greater optimism and a stronger sense of self-efficacy (Boniwell et al. 2010).

Stolarski et al. (2011) used yet another method to determine BTP in the respondents; they calculated a deviation coefficient of a given respondent's individual profile from an exemplary BTP (according to Zimbardo and Boyd 2008). The results they obtained showed that higher emotional intelligence is a predictor of smaller deviations from BTP, which is due to the fact that coping strategies and self-control of emotions determine BTP development. In their studies, the strongest predictor of the tendency to postpone gratification was the BTP coefficient calculated according to the proposed formula. It was interesting 
that none of the separated TP dimensions explained the tendency to postpone gratification. These results emphasise how important it is to include temporal profiles in studies instead of only analysing individual dimensions.

Zhang et al. (2013) compared three methods of measuring BTP, including a cluster analysis used by Boniwell et al. (2010), quartile deviations applied by Drake et al. (2008), and deviation of a BTP coefficient (DBTP) calculated on the basis of the formula for the deviation of an individual profile from exemplary one. The BTP most strongly correlated with well-being is when it was operationalised as DBTP (Stolarski et al. 2011). According to these authors, further studies in this area should focus on the relationship between BTP and other aspects of well-being and also check the influence of changes introduced to a TP structure on the measurement of BTP. Following these suggestions, in our investigation, we chose a positive orientation (PO) as an important indicator of well-being, as well as introducing a new component of BTP: active concentration on the present.

\subsection{Positive Orientation}

The development of positive psychology has allowed researchers to distinguish a whole range of phenomena that make up what we understand as well-being. Many of these phenomena show positive, moderate or high correlations with each other, which may indicate the presence of a higher-order factor explaining their common variability. While specific variables, such as self-esteem and optimism, help to further clarify the specific nature and significance of different areas of performance, a more general factor allows the explanation of the overriding dimension of well-being, thereby serving as a direct measure of general well-being. Caprara (2009) suggested that PO is such a factor. Caprara defines $\mathrm{PO}$ as a general tendency to respond to life experiences with a positive attitude (Alessandri et al. 2012; Caprara 2009; Caprara et al. 2010). From an empirical point of view, PO is a latent variable that explains the common variability of three variables used most frequently in studies on optimal functioning: self-esteem (measured with Self-Esteem Scale, SES, Rosenberg 1965), life satisfaction (Life Satisfaction Scale, SWLS, Diener et al. 1985) and optimism (Life Orientation Test, LOT-R; Scheier et al. 1994). Based on the overall score, a factor score is calculated, and that score is a measure of PO.

The use of $\mathrm{PO}$ as a measure of well-being allows the prediction of a significantly greater percentage of variability of other variables, when compared to models in which variables are predicted by means of self-esteem, optimism and life satisfaction treated as independent variables (Alessandri et al. 2012). Therefore, if well-being as such is the subject of the analysis, it is preferable to use PO rather than specific variables. In studies verifying the accuracy of this construct, PO is significantly positively correlated with hedonistic balance, the quality of interpersonal relationships and perceived health status (Caprara et al. 2010). Caprara et al. (2010) and Oles et al. (2013) also discovered a connection between PO and a sense of self-efficacy.

\section{Present Study}

The primary aim of the research presented in this article is to investigate the relationship between PO and TP in terms of Zimbardo and Boyd's work (1999), which is extended to a third form of the present TP: the active concentration on the present. Based on the studies presented above, we formulated the following hypotheses. 
Hypothesis 1 PO significantly correlates positively with a temporal profile characterised by deep concentration on positive past, moderate concentration on the future, moderate hedonistic concentration, poor fatalistic concentration, poor concentration on negative past, and deep active concentration on present.

Hypothesis 2 Canonical correlation between sets of variables that represent PO and TP would be stronger when Carpe Diem is included as additional present TP.

Hypothesis 3 Cluster analysis allows the identification of a cluster corresponding to $\mathrm{BTP}$, in which active concentration on the present intensity is high.

The relative importance of active concentration on the present to explain PO through the use of the BTP operationalisation method proposed by Stolarski et al. (2011, described in detail below) will also be examined. This method allows intensity specification of various temporal scales on the basis of theoretical assumptions regarding BTP. It also enables a calculation for each individual for just one variable that represents the deviation of this respondent's profile from the reference profile of BTP. Manipulating the intensity of particular temporal dimensions allows the development of several alternative coefficients of BTP, emphasising the different significance of various temporal perspectives: comparing models in which alternative temporal profiles are the PO predictor leads to choosing a profile that best explains PO. This part of the analysis is exploratory in nature.

\section{Method}

\subsection{Participants}

The study group consisted of 185 people (121 female; 64 male). The study was conducted in Poland on a group of 219 people, of whom 185 returned a set of completed questionnaires. The respondents were selected at random. Their ages were between 15 and 82 $(\mathrm{M}=37.02, \mathrm{SD}=15.9)$ and over $50 \%$ of the respondents were more than 35 years old at the time of the study. Nearly $50 \%$ of the respondents had a degree in economics, $16 \%$ in medicine and related sciences and $16 \%$ in pedagogy. Almost $70 \%$ of the respondents lived in a small town or in the country. Most of them received questionnaires directly from a psychologist or a psychologist's assistant. The others received questionnaires by mail.

\subsection{Measures}

\subsubsection{Time Perspective}

The ZTPI (Zimbardo and Boyd 1999) was used to measure five dimensions of TP. It is a self-descriptive test which consists of 56 items. It examines attitudes towards time: the past, the present and the future, and behaviours related to them. The respondent is asked to indicate how accurately or inaccurately each term describes the object by selecting an appropriate numerical response category (from $1=$ 'very untrue' to $5=$ 'very true'). The questionnaire consists of five scales. The Past-Positive scale (in Polish translation alpha $=.61$ ) examines concentration on a positively evaluated past (for example: 'It gives me pleasure to think about my past'). The Negative-Past scale (alpha $=.83$ ) refers to concentration on a negatively perceived past (for example: 'It's hard for me to forget unpleasant images from my youth'). The Present-Hedonistic scale (alpha $=.81$ ) measures 
hedonistic concentration on the present (for example: 'I take risks to put excitement in my life'). The Present-Fatalistic scale (alpha $=.72$ ) pertains to fatalistic orientation on the present (for example: 'Fate determines much in my life'). The Future scale (alpha $=.76$ ) examines concentration on a positively perceived future (for example: 'I believe that a person's day should be planned ahead each morning'). In our study, we used the Polish translation of the test (Sobol-Kwapinska et al. 2015) performed by Przepiorka and authorised by Zimbardo.

\subsubsection{Self-Esteem}

To measure self-esteem, an SES (Rosenberg 1965) was used and adapted to Polish circumstances (Dzwonkowska et al. 2008). It consists of 10 items (the Polish version of the scale alpha $=.83$ ). It measures the general level of a sense of one's self-worth as disclosed in the self-description and treated as a relatively constant personality trait. The respondent takes a stance on each of the statements on a four-point scale (from $1=$ 'strongly agree' to $4=$ 'strongly disagree'). This is a sample item: 'I feel that I have a number of good qualities'.

\subsubsection{Satisfaction with Life}

The SWLS by Diener et al. (1985) was used to measure a sense of satisfaction with life. The scale consists of five items (the Polish version of the scale alpha $=.85$; Juczynski 2012). This is a sample item: 'In most ways, my life is close to my ideal'. The respondent takes a stance on each of the statements on a seven-point scale (from $7=$ 'strongly agree' to $1=$ 'strongly disagree').

\subsubsection{Optimism}

Optimism was measured using the LOT-R by Scheier et al. (1994). The scale is composed of 10 items, while 4 of these are fillers and only 6 are relevant for the optimism score (the Polish version of the scale alpha $=.80$; Juczynski 2012). It measures expectations regarding the future and a general sense of optimism. This is a sample item: 'In uncertain times, I usually expect the best'. The respondent takes a stance on each of the statements on a five-point scale (from $4=$ 'strongly agree' to $0=$ 'strongly disagree').

\subsubsection{Active Present Perspective}

The Carpe Diem Scale by Sobol-Kwapinska (2009, 2013) was used to measure active concentration on the present $($ alpha $=.82)$. It consists of 10 items, for example: 'I appreciate the value of every moment of life'. The respondents are asked to indicate on a five-point scale the extent to which they agree with the given statements (from $1=$ 'completely disagree' to $5=$ 'completely agree'). The scale accuracy was determined by correlations with other measures of temporal attitudes (see Sobol-Kwapinska 2009, 2013). For example, the scale correlated significantly positively with the Telicity scale from Temporal Orientation Questionnaire AION-2000 by Nosal and Bajcar (2004), which measures concentration on goal achievement, significantly positively with the Purpose in Life scale (Crumbaugh and Maholick 1981), and with the Positive Affect scale from the Positive and Negative Affect Scale (PANAS; Watson et al. 1988). Moreover, the Carpe 
Diem Scale correlated moderately positively with the Present scale, also from the AION2000 questionnaire, measuring hedonistic and fatalistic concentration on the present (Sobol-Kwapinska 2013). High scores on the Carpe Diem Scale also linked with a positive evaluation of time in general (Sobol-Kwapinska and Nosal 2009).

\section{Results}

To check relations between PO and TP we performed pairwise correlations as well as canonical correlation analysis. Next we used cluster analysis to verify the third hypothesis that active concentration on the present is a significant component of the BTW profile, as it was defined by Zimbardo and Boyd (1999). Lastly, using Stolarski's et al. (2011) formula, we computed several alternative BTP indexes which differed in intensity of active and hedonic time perspectives. It allowed us to check which of the theoretically derived BTP versions predics $\mathrm{PO}$ best.

Table 1 shows descriptive statistics and correlations for variables in the study. The analysis of sex differences indicated only one variable, namely concentration on the future, for which men scored significantly lower than women $(\mathrm{t}=-2.68, p=.008)$. As far as other scales are concerned, the results were comparable for both men and women. Age was positively correlated only with future perspective, but this relation was very weak $(r=.15)$ what suggests that both neither the level of PO nor TP are age dependent. The correlations between individual PO components and TP dimensions are, in most cases, significant, although they range from weak to moderate. Self-esteem and optimism are weakly but significantly correlated with active concentration on the present $(\mathrm{r}=.24$ and $\mathrm{r}=.25$ respectively; significance for both is $p<.001$ ), while satisfaction with life is not related to active concentration on the present $(\mathrm{r}=.11, \mathrm{p} \mathrm{ns})$. The strongest negative relationship with PO components appeared in the case of negative past (Pearsons' $r$ equals from -.42 to $-.47, p<.001$ ), whereas for fatalistic present orientation (Pearsons'r equals from -.12 to $.06, \mathrm{p} \mathrm{ni})$, there were no relationships at all, consistent for all three components of PO. Only one.

\subsection{Canonical Correlations}

The pairwise correlations described above allow researchers only to capture the relationships between individual variables. Our study was designed to determine the extent to which PO allows the prediction of BTP. One way to answer this question is to use canonical correlations, which allows a researcher to check whether two groups of variables forming PO and TP correlate with each other as expected. For this purpose, we designed an analysis involving two sets of variables: one which included self-esteem, satisfaction with life and optimism, and another which consisted of the ZTPI individual scales. We also carried out an additional canonical analysis, which differed from the first only in one more variable, which we added to the set referring to TP, that is active concentration on the present.

For the analysis that did not include the Carpe Diem Scale, we obtained three functions with canonical correlations amounting to $.66, .29$ and .07 . The first and the second function were statistically significant, while the third function turned out to be irrelevant. Due to the small percentage of explained variance by the second function (it amounted to only $8 \%$ of variance unexplained by the first function), we decided to interpret only the first canonical 


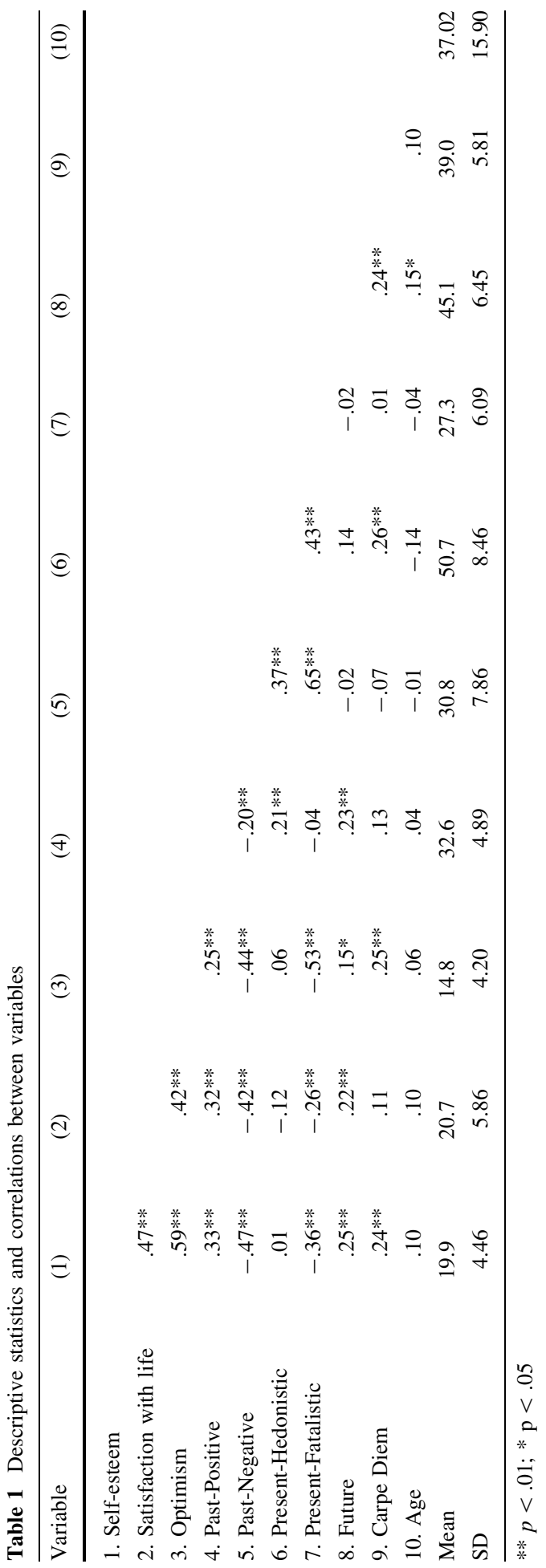


function in a further discussion. For the analysis that takes the Carpe Diem Scale into account we obtained three functions with canonical correlations amounting to $.67, .30$ and .07 . This time, only the first function was significant, whereas the others did not reach the threshold of significance (i.e., $p<.05$ ). Like in the previous analysis, we further interpret only the first canonical function.

Table 2 presents the factor loadings and redundancy for the first canonical function. $\mathrm{R}_{\mathrm{c}}^{2}$ indicates that both canonical variables share $43 \%$ of their variance (45\% after taking the Carpe Diem Scale into account). The canonical factor loadings for self-esteem, satisfaction with life and optimism are similar to those obtained in other studies (e.g., Oles et al. 2013). This allows the interpretation of this canonical variable as representing PO. The PO latent variable explains $66 \%$ of the variance shared by self-esteem, satisfaction with life and optimism. These three variables are also moderately correlated with the second canonical variable (see cross-loadings for the Set 1 in the Table 1) which explains $29 \%$ of their common variance.

The factor loadings for the second set of variables (temporal) indicate that this canonical variable, which explains $27 \%$ of variance shared by ZTPI scales, mainly reflects low scores in Past-Negative and Present-Fatalistic, high in Past-Positive and moderate in Future. What is interesting, Past-Negative and Present-Fatalistic scales are also significantly correlated with PO latent variable, as cross-loadings for the Set 2 show. The structure of the factor loadings reminds us of the structure of BTP as postulated by Zimbardo et al. (Boniwell and Zimbardo 2004; Zimbardo and Boyd 2008), with the exception of the score in Present-Hedonistic scale. Present-Hedonistic seems to have no relevance to the interpretation of the temporal latent variable correlated with PO. The canonical variable, which is the result of the empirical adjustment of a model to data, indicates that PO predicts a set of temporal variables that is dominated primarily by low Past-Negative and Present-Fatalistic. In other words, while BTP stresses the importance of positive aspects of TP, empirical analysis emphasizes a low intensity of negative dimensions. An important difference between the theory and the results of canonical analysis is the Present-Hedonistic insignificance for the interpretation of the latter.

What is important, active concentration on the present proves to be a meaningful component of the BTP-it is moderately (.38) correlated with BTP latent variable. Model that includes Carpe Diem Scale as a BTP component explains more variance $\left(\mathrm{R}_{\mathrm{c}}^{2}=.45\right)$ compared to the model that represents only ZTPI scales $\left(\mathrm{R}_{\mathrm{c}}^{2}=.43\right)$.

Table 2 Factor loadings and redundancy for the first canonical function

Values displayed in brackets refer to the model not including active perspective on the present

\begin{tabular}{lcc}
\hline & Loadings & Cross-loadings \\
\hline Set 1: positive orientation variables & \\
Self-esteem & $.90(.89)$ & $.60(.59)$ \\
Life Satisfaction & $.72(.73)$ & $.48(.48)$ \\
Optimism & $.81(.80)$ & $.54(.53)$ \\
Set 2: temporal variables & & $.37(.37)$ \\
Past-positive & $.55(.56)$ & $-.55(-.55)$ \\
Past-negative & $-.82(.-83)$ & $-.01(-.01)$ \\
Present-hedonistic & $-.01(-.02)$ & $-.40(-.40)$ \\
Present-fatalistic & $-.60(-.61)$ & $.26(.26)$ \\
Future & $.39(.40)$ & .26 \\
Carpe Diem & .38 & \\
\hline
\end{tabular}




\subsection{Cluster Analysis}

Canonical correlations help trace the extent to which variables related to PO correlate with TP dimensions. They do not allow, however, for the comparison of people with different TP profiles in terms of specific variables. To separate respondents with different TP profiles, we applied a hierarchical cluster analysis, carried out with the use of Ward's method and Euclidean squared distances. Following Zhang et al. (2013), we conducted a cluster analysis, assuming a division into groups of two, three, four and five possible clusters so as to avoid a situation in which the criterion for separating the number of clusters will reduce the chance of finding a group of people with a typical BTP profile. We conducted the cluster analysis for the set of variables that consisted of the standardised results of the ZTPI scales increased by the Carpe Diem Scale.

This cluster analysis allowed the identification of people with BTP, assuming that the Carpe Diem Scale is considered to be an alternative to Present-Hedonistic concentration on the present. When three clusters were separated, one of the groups was characterised by high scores in Past-Positive and Future, low scores in the Past-Negative and PresentFatalistic and moderately high intensity on the Carpe Diem Scale. The scores on the Present-Hedonistic scale in this cluster were around the average. Other clusters were typical for people with low intensity of most of the TP dimensions (except for PastNegative and Present-Fatalistic, which were at average level) and those with very high scores in the Past-Negative and Present-Fatalistic, high Present-Hedonistic, moderately high Future and Carpe Diem and low Past-Positive scores. The groups were different in terms of PO intensity, $\mathrm{F}(2,180)=32.87, p<.001$, ETA2 $=.27$, and post hoc tests (with the Bonfferoni correction) demonstrated a significant difference between the BTP group and the other two clusters $(p<.001)$, which, in turn, did not differ significantly from each other $(p=.34)$.

\subsection{BTP Theoretical Operationalisation}

Both canonical and cluster analysis are exploratory statistical methods and we applied them here to determine which set of variables representing different TP profiles would be fully predicted by PO. The analyses we carried out indicated Present-Hedonistic to be the scale which is not significantly correlated with optimal functioning as measured by PO. They pointed, however, to Carpe Diem as a variable significantly correlated with wellbeing. In addition to these methods of data analysis, it is possible to operationalise BTP based on theoretical assumptions. There are two attempts of this kind in the literature: (1) separating people with a temporal profile corresponding to the reference profile and comparing their level of functioning, and (2) formulating a numerical coefficient to measure the deviation of a given person's profile from the reference profile and correlating it with measures of well-being. The first of these approaches, proposed by Drake et al. (2008), raises many problems, both from statistical and interpretative points of view. The separated groups are usually not large enough and constitute about $5 \%$ of the study sample. In addition, the criteria for separating individuals are also more dependent on the description of a given sample (the cut-off point $=$ the $33 \mathrm{rd}$ percentile in a given scale) rather than reflecting theoretical assumptions.

The second method of theoretical operationalisation of BTP, proposed by Stolarski et al. (2011), allows researchers to obtain the direct BTP coefficient calculated on the basis of theoretical assumptions. Studies conducted by Zhang et al. (2013) showed that BTP 
correlates most with the well-being measures when it is operationalised as DBTP. For the calculation of the DBTP coefficient, the following formula is used:

\section{$D B T P 1$}

$$
=\sqrt{(o P N-e P N)^{2}+(o P P-e P P)^{2}+(o P F-e P F)^{2}+(o P H-e P H)^{2}+(o F U-e F U)^{2}}
$$

where $o P N-e P N$ is the difference between optimal intensity of the Past-Negative scale for BTP (according to Zimbardo's concept) and the empirical score on this scale obtained from a given participant. Further segments of the equation correspond to successive ZTPI scales. In the present study, we created five alternative coefficients that include both ZTPI scales and the score on the Carpe Diem Scale or omit the Present-Hedonistic scale in the equation, replacing it with the Carpe Diem Scale. Following Stolarski et al. (2011) who, in turn, referred to Zimbardo and Boyd's proposals (cf. www.thetimeparadox.com/surveys), the following intensity of individual scales (optimal of BTP) were adopted: high PastPositive (4.60); moderately high Present-Hedonistic (3.90); moderately high Future (4.00); low Past-Negative (1.95), and low Present-Fatalistic (1.50). In addition, we decided that the Carpe Diem Scale intensity should be, as in the case of Present-Hedonistic, 3.90 or higher, that is 4.60, as in the case of Past-Positive. The formula expresses the deviation of the optimal profile from the profile obtained by the respondent: the higher the DBTP coefficient, the further from the optimal TP it is for a single person. A zero score in DBTP indicates perfectly balanced temporal perspective, according to the criteria.

In order to verify the hypothesis, according to which active concentration on the present is a better BTP indicator than Present-Hedonistic, three regression analyses were conducted, in which PO was the variable criterion, while consecutive DBTP coefficients with indices from one to three were the predictors. Such a solution was chosen not because of theoretical assumptions that suggest rather a reverse path direction, but for practical reasons, to compare the models with the same dependent variable to each other. It was assumed that $\mathrm{DBTP}_{1}$ reflects the BTP coefficient calculated only for five ZTPI scales; $\mathrm{DBTP}_{2}$ includes all ZTPI scales together with the Carpe Diem Scale (high intensity in the profile-4.60), and $\mathrm{DBTP}_{3}$ includes all ZTPI scales together with the Carpe Diem Scale (moderately high intensity in the profile-3.90).

The PO index was created by means of a factor analysis conducted for the summary scores of the SES, SWLS and LOT-R. Both the Kaiser criterion and the scree plot pointed to one important factor explaining $66 \%$ of the common variability of the above-mentioned variables. The factor loading for self-esteem was the highest (.85), was slightly lower for optimism (.83), and was the lowest for satisfaction with life (.76), although still very high.

Table 3 shows the results of the three regression models ${ }^{1}$ in which the DBTP alternative coefficients were the PO predictors. In order to select the model that best explains the variables set, the Akaike information criterion (AIC) was used. According to the AIC, the model which includes the $\mathrm{DBTP}_{2}$ coefficient, that is, the model including both moderate hedonistic concentration intensity and high active concentration on the present intensity turns out to be the least deviated from the real model compared to the other models. The rest of the AIC coefficients differ from the AIC coefficient for $\mathrm{DBTP}_{2}$ model by more than ten. Such a difference (although all models are adjusted to data) allows the rejection thereof, as models were significantly worse at explaining data: based on AIC weights, it is

\footnotetext{
1 The age was not included in the models as a covariant because it was not correlated either with positive orientation factor $(\mathrm{r}=.10, \mathrm{p} \mathrm{ns})$ or any version of DBTP index ( $\mathrm{r}$ equals from -.08 to $-.03, \mathrm{p} \mathrm{ns}$ ).
} 
Table 3 Comparison of five regression models with optimal-empirical temporal profiles discrepancy as a predictor of positive orientation

\begin{tabular}{lcccccccrc}
\hline Predictor & $\mathrm{F}(1,181)$ & $\mathrm{B}$ & $\mathrm{SE}$ & Beta & $\mathrm{t}$ & $\mathrm{R}^{2}$ & AIC & $\Delta$ AIC & AIC weight \\
\hline DBTP $_{1}$ & 106.38 & -.80 & .08 & -.61 & -10.31 & .37 & 439.72 & 12.09 & .002 \\
$\mathrm{DBTP}_{2}$ & 126.01 & -.87 & .08 & -.64 & -11.22 & .41 & 427.63 & .00 & .997 \\
$\mathrm{DBTP}_{3}$ & 89.44 & -1.02 & .11 & -.58 & -9.46 & .33 & 450.84 & 23.21 & $<.001$ \\
\hline
\end{tabular}

possible to determine a probability of $99.7 \%$ that the second model is the best, despite the sample (Burnham and Anderson 2002). The model in which BTP takes into account high intensity of active concentration on the present explains $41 \%$ of the PO variation, which proves large effect size.

In conclusion, the data analysis confirms significant correlations between $\mathrm{PO}$ and the set of temporal variables postulated by Zimbardo (Boniwell and Zimbardo 2004; Zimbardo and Boyd 1999, 2008). An important difference is the minor significance of hedonistic concentration on the present (Present-Hedonistic) in explaining PO and major significance of active concentration on the present.

\section{Discussion}

This study was aimed at verifying hypotheses about the relationships between TP according to Zimbardo and Boyd $(1999,2008)$ and PO according to Caprara (2009). We postulated that PO correlates with BTP, i.e., with deep concentration on a positively evaluated past, moderately deep concentration on the future, moderately deep hedonistic concentration on the present, poor fatalistic concentration on the present, and poor concentration on a negatively assessed past. We also introduced an additional kind of concentration on the present, namely an active Carpe Diem concentration (Sobol-Kwapinska 2009, 2013), which, according to the hypothesis, was to fit the frames of the BTP profile and positively correlate with PO. In order to verify the above postulates, we used the Polish version of the ZTPI, increased by the Carpe Diem Scale.

The results we obtained partially confirm the postulated relationship between PO and BTP. Frequent concentration on a positively perceived past combined with moderately deep concentration on the future, poor fatalistic concentration and poor concentration on a negatively assessed past, is strongly and positively correlated with PO. Only the hedonistic concentration on the present (Present-Hedonistic) was neither included in the set of temporal variables related to $\mathrm{PO}$, nor was significantly intensified in the cluster characterised by a set of scales resembling BTP. Our results, aside from the Present-Hedonistic scale, largely support the BTP theory proposed by Zimbardo and Boyd (2008). Drake et al. (2008) discovered that hedonistic concentration on the present correlated negatively with subjective well-being. The results of a study published recently by Sircova et al. (2015), have shown that the BTP profiled and identified in a multicultural sample by cluster analysis includes a moderately low level of hedonistic perspective. In studies conducted by Wiberg et al. (2012), it was an average, not moderately high, Present-Hedonistic score that made up the balanced TP profile. On the other side, in studies undertaken by Boniwell et al. (2010) and by Zhang et al. (2013) Present-Hedonistic positively correlated with the wellbeing measures. The ambiguity of the results regarding the Present-Hedonistic scale 
significance for the BTP profile can be explained by the considerable instability of correlation between this scale and various indicators of well-being. In other words, the strength and direction of a possibly weak relationship may be seriously affected by the selection of respondents to join the research sample. In our case, the unique nature of Polish culture may have additional meaning. Perhaps the way the Polish people understand and express hedonistic perspective is different to that of Western cultures, for instance, Americans. The results of studies conducted by Sobol-Kwapinska (2014) confirm this assumption, as they suggest that hedonistic concentration on the present verified with the use of the Polish version of the ZTPI is a many-sided variable. For example, a tendency to carry out risky behaviours, which in the original version of the ZTPI belongs to PresentHedonistic, in Polish version correlated more with the fatalistic perspective rather than with Present-Hedonistic. This suggests an interesting interpretation that Americans take risks mainly for pleasure, while Polish people do so because of a conviction that they cannot affect reality. Similarly, the energy and activity which, in the original version, belonged to the Present-Hedonistic scale, correlated more with concentration on the future in the Polish version. On this basis, we may speculate that perhaps the hedonism of Polish people might have fewer positive connotations when compared to Americans. Perhaps the Polish people give themselves permission to enjoy life less often than Americans. This would be a very interesting issue to explore in future studies. Cultural factors appear to be vitally important in explaining the variance of results obtained in different countries, especially if we pay attention to the fact that Drake et al. (2008) conducted their studies among the Scots, Boniwell et al. (2010) described the findings regarding Britons and Russians, Wiberg et al. (2012) studied the Swedes, while Zhang et al. (2013) focused their academic interests on Americans. Moreover, in the studies conducted by Boniwell et al. (2010) positive correlation between hedonism (Present-Hedonistic) and SWB measures was much weaker in the group of Russians than in the group of Britons.

Active concentration on the present significantly explained BTP, which confirms our hypothesis. In the canonical analysis, the Carpe Diem Scale was of moderate importance for the interpretation of the set of variables that affects PO the most. Similarly, the cluster analysis allowed for the separation of a group of individuals having a profile corresponding to the BTP, where active concentration on the present intensity was, in contrast to hedonistic concentration, moderately high. Calculating the DBTP coefficients reflecting the average deviation of a given person's profile from the reference profiles allowed us to clarify the role that these two types of concentration on the present play in explaining PO. Comparing different models suggests that the profile best explaining PO contains both the Present-Hedonistic scale of moderately high intensity and the Carpe Diem Scale of high intensity, equal to the Past-Positive intensity.

An important novelty in this study is the highlighting of active concentration on the present as a temporal perspective important to well-being. Our results suggest the correctness of expanding BTP by an additional dimension, namely active concentration on the present. This is in line with postulates of many theories, in which the importance of balance between the quest for pleasure and comfort, the development of one's own abilities and achievement of important goals is stressed (e.g., Ryan and Deci 2001).

Our results support the main idea behind the formulation of the BTP concept. According to this idea, high intensity of Past-Positive and Future and low intensity of Past-Negative and Present-Fatalistic favours a flexible adaptation to different conditions of life and effective performance of tasks (Boniwell and Zimbardo 2004). Thanks to flexibility, people are able to change their attitudes towards time depending on the requirements of a given situation. In other words, when, for example, people spend time with friends, they concentrate on common 
history; when they rest, they focus on the present, free from a feeling of guilt that they are not working; when they work, they work with a view to future consequences of their present behaviours and draw from past experiences (Boniwell and Zimbardo 2004; Zimbardo and Boyd 1999, 2008). Therefore, BTP allows people to freely go on mental travels in time, one of the basic human adaptabilities. Balanced temporal perspective, expanded by active concentration on the present, allows people to both 'travel in time' and also to anchor to present reality. For example, in order for work to give satisfaction and pleasure, active concentration on the present is necessary; such concentration allows people to experience mental state of the flow, in which a person performing some activity is fully immersed in this activity (cf. SobolKwapinska 2009, 2013). Active concentration on the present is also important in crisis situations. The results of studies conducted by Epel et al. (1999) among homeless people show that concentration on the present is an optimal attitude towards time in a situation of dealing with a problem (acute crisis). While concentration on the future correlates with a greater sense of self-efficacy and optimism, concentration on the present may facilitate openness to seeking quick solutions to current problems.

With regard to the limitations of our studies, it is important to stress that, due to the fact that the studies are correlative in nature, it is difficult to interpret the relationships we discovered in terms of cause and effect. In addition, we did not consider the past of individual respondents (for example, the possible presence of significant negative experiences). Arguably, events from the past may affect the scores obtained in the ZTPI, as suggested by Drake et al. (2008). In subsequent studies, taking into consideration such events as possible co-variants and checking BTP adaptive significance not in everyday life, but also in specific life situations, such as illness, suffering pain, grief, etc., would be worthwhile. The impact of cultural factors on the TP structure would be a highly important issue to explore in further studies as well. It would be also interesting to conduct an investigation into subgroups separated by age of the subjects. Differences in the relationship can be expected between PO and TP, depending on the age of the respondents (see Carstensen et al. 1999).

Regarding another limitation of this study it is also worth noting that a focus on the negatively evaluated future has not been studied. Carelli et al. (2011) emphasised the need to consider this kind of TP in research.The use of the scale proposed by them might be fruitful in the future research.

The results of our studies may have practical applications. An insight into what thoughts and feelings a patient has about the past, the present and the future may be a good starting point in therapy. A TP that is too narrow and rigid (i.e., unbalanced) may turn out to be the source of a patient's problems. Using the knowledge resulting from the studies on BTP in therapeutic work with depressive patients may be particularly useful because PO is considered to be the opposite of depressive cognitive triad. People suffering from depression are usually concentrating on negative past and fatalistic present and transfer this approach to the future. A therapy programme should therefore be based on teaching such persons how to deal with negative experiences by discovering positive aspects hidden in them, concentrating on the 'here and now' and planning a positive future (cf. Zimbardo et al. 2012).

\section{Summary}

In our study, we investigated the relationship between PO and TP as outlined by Zimbardo and Boyd (1999) and extended by the Carpe Diem Scale. We took on such studies to check whether the theoretical concept of BTP according to Zimbardo and Boyd (2008), referred 
to as a generally positive, optimal attitude towards time, would indeed strongly correlate with an overall positive attitude towards life. The results we obtained allow us to formulate an affirmative and convincing answer to this question and may provide a starting point to design strategies to help people improve their level of functioning. When working on a more balanced TP, it is possible to improve the functioning of an individual, regardless of the intensity of this person's general innate tendency to make positive evaluations regarding themselves, life and the future.

Moreover, we justified in our studies the value of expanding the five dimension TP proposed by Zimbardo to include active concentration on the present in order to capture the present perspective in a more holistic way. Furthermore, we conducted our studies among adults who are not students. This is important in as much as the vast majority of temporal studies have been conducted with the participation of students. Meanwhile Ryack (2012), like many other researchers, draws attention to the fact that TP dimensions depend on the group on which such studies are conducted.

Conflict of interest The authors declare there are no potential conflicts of interest with respect to the research, authorship, and/or publication of this article. The authors disclosed receipt of the following financial support for the research, authorship, and/or publication of this article: preparation of this manuscript was supported by the grant 'Positive orientation-structure and functions' $1 / 6-3-8-27$ and the grant 'Time perspective, postoperative pain perception and coping strategies in pain' 2013/09/B/HS6/02785 from the National Science Centre.

Open Access This article is distributed under the terms of the Creative Commons Attribution 4.0 International License (http://creativecommons.org/licenses/by/4.0/), which permits unrestricted use, distribution, and reproduction in any medium, provided you give appropriate credit to the original author(s) and the source, provide a link to the Creative Commons license, and indicate if changes were made.

\section{References}

Alessandri, G., Caprara, G. V., \& Tisak, J. (2012). The unique contribution of positive orientation to optimal functioning: Farther explorations. European Psychologists, 17, 44-54.

Barber, L. K., Munz, D. C., Bagsby, P. G., \& Grawitch, M. J. (2009). When does time perspective matter? Self-control as a moderator between time perspective and academic achievement. Personality and Individual Differences, 46, 250-253.

Boniwell, I., Osin, E., Linley, P., \& Ivanchenko, G. (2010). A question of balance: Examining relationships between time perspective and measures of well-being in the British and Russian samples. Journal of Positive Psychology, 5, 24-40.

Boniwell, I., \& Zimbardo, P. (2004). Balancing time perspective in pursuit of optimal functioning. In P. A. Linley \& S. Joseph (Eds.), Positive psychology in practice (pp. 165-178). Hoboken, NJ: Wiley.

Boyd, J. N., \& Zimbardo, P. G. (2005). Time perspective, health and risk taking. In A. Strathman \& J. Joireman (Eds.), Understanding behavior in the context of time (pp. 85-107). London: Lawrence E.

Bryant, F. B., Smart, C. M., \& King, S. (2005). Using the past to enhance the present: Boosting happiness through positive reminiscence. Journal of Happiness Studies, 6, 227-260.

Burnham, K. P., \& Anderson, D. R. (2002). Model selection and multimodel inference. New York, NY: Springer.

Caprara, G. V. (2009). Positive orientation: Turning potentials into optimal functioning. The Bulletin of the European Health Psychologist, 11, 46-48.

Caprara, G. V., Alessandri, G., \& Barbaranelli, C. (2010a). Optimal functioning: The contribution of selfefficacy beliefs to positive orientation. Psychotherapy and Psychosomatics, 79, 328-333.

Caprara, G. V., Steca, P., Alessandri, G., Abela, J. R., \& McWhinnie, C. D. (2010b). Positive orientation: Exploration on what is common to life satisfaction, self-esteem, and optimism. Epidemiology and Psychiatric Sciences, 19, 63-71.

Carelli, M., Wiberg, B., \& Wiberg, M. (2011). Development and construct validation of the Swedish Zimbardo Time Perspective Inventory. European Journal of Psychological Assessment, 27, 220-227. 
Carstensen, L. L., Isaacowith, D. M., \& Charles, S. T. (1999). Taking time seriously: A theory of socioemotional selectivity. American Psychologist, 54(3), 165-181.

Crumbaugh, J. C., \& Maholick, L. T. (1981). Manual of instructions for purpose-in-life test. Murfreesboro, TN: Psychometric Affiliates.

D’Alessio, M., Guarino, A., de Pascalis, V., \& Zimbardo, P. G. (2003). Testing Zimbardo's Stanford Time Perspective Inventory-Short Form: An Italian study. Time \& Society, 12, 333-347.

Daugherty, J. R., \& Brase, G. L. (2010). Taking time to be healthy: Predicting health behaviors with delay discounting and time perspective. Personality and Individual Differences, 48, 202-207.

Diener, E., Emmons, R., Larsen, J., \& Griffin, S. (1985). The satisfaction with life scale. Journal of Personality Assessment, 49, 71-75.

Drake, L., Duncan, E., Sutherland, F., Abernethy, C., \& Henry, C. (2008). Time perspective and correlates of well-being. Time and Society, 17, 47-61.

Dzwonkowska, I., Lachowicz-Tabaczek, K., \& Laguna, M. (2008). Samoocena i jej pomiar Skala samooceny SES M. Rosenberga [Self-esteem and its measure]. Warszawa: Pracownia Testow Psychologicznych PTP.

Epel, E. S., Bandura, A., \& Zimbardo, P. G. (1999). Escaping homelessness: The influences of self-efficacy and time perspective on coping with homelessness. Journal of Applied Social Psychology, 29, 575-596.

Juczynski, Z. (2012). Narzedzia pomiaru w promocji i psychologii zdrowia [Measuring tool in the promotion and health psychology]. Warszawa: Pracownia Testów Psychologicznych PTP.

Keough, K. A., Zimbardo, P. G., \& Boyd, J. N. (1999). Who's smoking, drinking, and using drugs? Time perspective as a predictor of substance use. Journal of Basic and Applied Social Psychology, 21, $149-164$.

Klingemann, H. (2001). The time game: Temporal perspectives of patients and staff in alcohol and drug treatment. Time \& Society, 10, 303-328.

Lennings, C. J., Burns, A. M., \& Cooney, G. (1998). Profiles of time perspective and personality: Developmental considerations. The Journal of Psychology, 182, 629-641.

Lyubomirsky, S., \& Nolens-Hoeksema, S. (1995). Effects of self-focused rumination on negative thinking and interpersonal problem solving. Journal of Personality and Social Psychology, 69, 176.

Nolen-Hoeksema, S., \& Morrow, J. (1993). Effects of rumination and distraction on naturally occurring depressed mood. Cognition and Emotion, 7, 561-570.

Nosal, C. S., \& Bajcar, B. (2004). Czas psychologiczny: wymiary, struktura, konsekwencje [Psychological time: dimensions, structure, consequences]. Warszawa: Wydawnictwo Instytutu Psychologii PAN.

Nuttin, J., \& Lens, W. (1985). Future time perspective and motivation: Theory and research method. Leuven: Leuven University Press.

Oles, P. K., Alessandri, G., Oles, M., Bak, W., Jankowski, T., Laguna, M., \& Caprara, G. V. (2013). Positive orientation and generalized self-efficacy. Studia Psychologica, 55(1), 47-59.

Rosenberg, M. (1965). Society and the adolescent self-image. Princeton, NJ: Princeton University Press.

Rothspan, S., \& Read, S. J. (1996). Present versus future time perspective and HIV risk among heterosexual college students. Health Psychology, 15, 131-134.

Ryack, K. (2012). Evidence that time perspective factors depend on the group: Factor analyses of the CFC and ZTPI scales with professional financial advisors. Personality and Individual Differences, 52, 723-727.

Ryan, R. M., \& Deci, E. D. (2001). On happiness and human potentials: A review of research on hedonic and eudaimonic well-being. Annual Review of Psychology, 52, 141-166.

Scheier, M. F., Carver, C. S., \& Bridges, M. W. (1994). Distinguishing optimism from neuroticism (and trait anxiety, self-mastery, and self-esteem): A reevaluation of the Life Orientation Test. Journal of Personality and Social Psychology, 67, 1063-1078.

Shostrom, E. L. (1974). Manual for the personal orientation inventory. San Diego, CA: Educational and Industrial Testing Service.

Sircova, A., Van de Vijver, F. J. R., Osin, E., Milfont, T. L., Fieulaine, N., Kislali-Erginbilgic, A., et al. (2015). Time perspective profiles and cultures. In M. Stolarski, N. Fieulaine, \& W. van Beek (Eds.), Time perspective theory; review, research and application; essays in honor of Philip G. Zimbardo (pp. 169-187). Switzerland: Springer.

Sobol-Kwapinska, M. (2009). Forms of present time orientation and satisfaction with life in the context of attitudes toward past and future. Social Behavior and Personality, 37, 433-444.

Sobol-Kwapinska, M. (2013). Hedonism, fatalism and "Carpe Diem": Profiles of attitudes towards the present time. Time \& Society, 22(3), 371-390. 
Sobol-Kwapinska, M. (2014). Carpe Diem, Zimbardo time perspective and positive orientation. Paper presented at the 2nd international conference on time perspective, Warszawa.

Sobol-Kwapinska, M., \& Nosal, C. S. (2009). How does one conceive time? Measurement by means of Time Metaphors Questionnaire. Polish Psychological Bulletin, 40, 1-7.

Sobol-Kwapinska, M., Przepiorka, A., \& Zimbardo, P. G. (2015). Structure of time perspective in Poland: Validation and extension of the Zimbardo Time Perspective Inventory across the ages. European Journal of Psychological Assessment (in review).

Stolarski, M., Bitner, J., \& Zimbardo, P. G. (2011). Time perspective, emotional intelligence and discounting of delayed awards. Time \& Society, 20(3), 346-363.

Strathman, A., Gleicher, F., Boninger, D. S., \& Edwards, C. S. (1994). The consideration of future consequences: Weighing immediate and distant outcomes of behavior. Journal of Personality and Social Psychology, 66, 742-752.

Watson, D., Clark, A., \& Tellegen, A. (1988). Development and validation of brief measures of positive and negative affect: The PANAS Scales. Journal of Personality and Social Psychology, 54(6), 1063-1070.

Wiberg, M., Sircova, A., Wiberg, B., \& Carelli, M. G. (2012). Operationalizing balanced time perspective in a Swedish sample. The International Journal of Educational and Psychological Assessment, 12(1), 95-107.

Wills, T. A., Sandy, J. M., Yaeger, A. M., Cleary, S. D., \& Shinar, O. (2001). Coping dimensions, life stress, and adolescent substance use: A latent growth analysis. Journal of Abnormal Psychology, 110, 309-323.

Zaleski, Z., Cycon, A., \& Kurc, A. (2001). Future time perspective and subjective well-being in adolescent samples. In P. Schmuck \& K. M. Sheldon (Eds.), Life goals and well-being: Towards a positive psychology of human striving (pp. 68-95). Ashland: Hogrefe \& Huber.

Zhang, J. W., Howell, R. T., \& Stolarski, M. (2013). Comparing three methods to measure a balanced time perspective: The relationship between a balanced time perspective and a subjective well-being. Journal of Happiness Studies, 14, 169-184.

Zimbardo, P. G., \& Boyd, J. N. (1999). Putting time in perspective: A valid reliable individual differences metric. Journal of Personality and Social Psychology, 77, 1271-1288.

Zimbardo, P. G., \& Boyd, J. N. (2008). The time paradox: The new psychology of time that will change your life. New York, NY: The Free Press.

Zimbardo, P. G., Keough, K. A., \& Boyd, J. N. (1997). Present time perspective as a predictor of risky driving. Personality and Individual Differences, 23(6), 1007-1023.

Zimbardo, P. G., Sword, R. M., \& Sword, R. K. (2012). The time cure: Overcoming PTSD with the new psychology of time perspective therapy. San Francisco, CA: Jossey-Bass. 